EPJ manuscript No.

(will be inserted by the editor)

\title{
Electromagnetic form factors of bound nucleons revisited
}

\author{
Ulughbek T. Yakhshiev ${ }^{1,2}$, Ulf-G. Meißner ${ }^{1}$ a , and Andreas Wirzba ${ }^{1}$ \\ 1 Institut für Kernphysik (Theorie), Forschungszentrum Jülich, D-52425 Jülich, Germany \\ 2 Theoretical Physics Department and Institute of Applied Physics, National University of Uzbekistan, Tashkent-174, \\ Uzbekistan \\ Received: date / Revised version: date
}

\begin{abstract}
We investigate the possible modifications of the nucleons' electromagnetic form factors in the framework of a modified Skyrme model allowing for nucleon deformation and using realistic nuclear mass distributions. We show that such effects are small in light nuclei.
\end{abstract}

PACS. 13.40.Gp - 12.39.Fe $-21.65 .+\mathrm{f}$

\section{Introduction}

It is firmly believed that hadron properties must undergo changes in a baryon-rich or hot environment. Many data from quasi-elastic or deep inelastic electron scattering off nucleons or nuclei, deeply bound pionic atoms and from heavy-ion reactions point towards such effects, but despite a tremendous amount of effort no clear theoretical picture concerning such scale changes has emerged It is therefore mandatory to study realistic models of the nucleon (or other hadrons) and to investigate how certain properties will change at finite baryon density. A popular class of such models are based on the Skyrme Lagrangian and variations thereof, for essentially three reasons: First, such models have proven to lead to a fairly good description of a wide variety of nucleon properties in free space and secondly, being based on non-linearly interacting pion fields, they are built from the degrees of freedom directly related to the spontaneous chiral symmetry breaking that QCD is supposed to undergo. Third, in contrast to (most) quark models, the pion cloud contribution of the nucleon is naturally taken into account. In earlier studies rather large medium modifications were found, based on the assumption of a constant background density (homogeneous nuclear matter), see e.g. Refs. [1, 2, 3, 4, 5, 6, 7]. In Ref. [8] we had formulated a more realistic version of such an approach, taking into account not only realistic distributions of baryon density within light, medium and heavy nuclei, but also allowing the nucleons to deform under the influence of the ensuing baryon density gradients. In particular, it was shown that the popular concept of a uniform

\footnotetext{
a Address after January $1^{\text {st }}, 2003$ : Helmholtz Institut für Strahlen- und Kernphysik (Theorie), Universität Bonn, Nußallee 14-16, D-53115 Bonn, Germany.

1 For example, the important question of disentangling genuine scale changes from "standard" many-body effects remains to be solved in a concise manner.
}

size modification (nucleon swelling or shrinking) cannot be maintained in such a picture, i.e. that the influence of the nuclear medium and the response of the nucleon to it is very probe dependent. In particular, the scale changes of the isoscalar and isovector electromagnetic charge distributions depend on the direction considered (there is an axial symmetry around the direction from the center of the nucleus to the center of the nucleon at some distance $R$ ) giving rise to a small intrinsic quadrupole moment. While the proton's shape changes from oblate to a prolate shape as it is moved toward the surface of the nucleus, the behaviour of the neutron is just the opposite. It is therefore of interest to reconsider the possible modifications of the nucleons' electromagnetic form factors, triggered also by the experimental fact that in the absence of neutron targets one has to use light nuclei like deuterium or ${ }^{3} \mathrm{He}$ to determine the neutron form factors. This will be the topic of the present paper, where we study these form factors inside ${ }^{4} \mathrm{He}$, which is the lightest nucleus that can be approximated by a continuous matter distribution (for a similar calculation including nuclear shell effects, see [5]). Finally, we note that a more systematic approach to tackle these questions based on effective field theory is not yet available for nucleons and only in its infancy for pions; for an attempt see e.g. [9] (and references therein).

Our work is organized as follows. In Section 2 we briefly review the underlying modified Skyrme model and give formulae for the electromagnetic form factors for the case of finite baryon density. In Section 3, we show and discuss the results for the specific case of ${ }^{4} \mathrm{He}$. We refrain from discussing other nuclei as done e.g. in Refs. [5. [8] since these results are genuine and can be scaled easily. We conclude with a short summary in Section 4 . 


\section{Brief description of the model}

\subsection{Lagrangian}

Our starting point is a modified Skyrme Lagrangian in the nuclear medium [7]

$$
\begin{aligned}
\mathcal{L}=\frac{F_{\pi}^{2}}{16} & \operatorname{Tr}\left(\frac{\partial U}{\partial t}\right)\left(\frac{\partial U^{\dagger}}{\partial t}\right)-\frac{F_{\pi}^{2}}{16} \alpha_{p}(\boldsymbol{r}) \operatorname{Tr}(\boldsymbol{\nabla} U)\left(\boldsymbol{\nabla} U^{\dagger}\right) \\
+ & \frac{1}{32 e^{2}} \operatorname{Tr}\left[L_{\mu}, L^{\nu}\right]^{2}+\frac{F_{\pi}^{2} m_{\pi}^{2}}{16} \alpha_{s}(\boldsymbol{r}) \operatorname{Tr}\left[U+U^{\dagger}-2\right]
\end{aligned}
$$

with $F_{\pi}$ the weak pion decay constant, $m_{\pi}$ the pion mass, $U(\boldsymbol{r})$ parametrizes the pion fields and $L_{\mu}=U^{\dagger} \partial_{\mu} U$ is the left-handed current. The Skyrme parameter $e$ (together with the pion decay constant) will be determined from fitting the masses of the nucleon and the $\Delta(1232)$. Its value can be understood in terms of rho meson exchange. We use a deformed ansatz for the pion fields when the Skyrmion is located at some distance $\boldsymbol{R}$ from the center of the nucleus ${ }^{2}$

$$
\begin{aligned}
U(\boldsymbol{r}) & =\exp [i \boldsymbol{\tau} \cdot \boldsymbol{N}(\Theta(\theta), \varphi) F(r, \theta)], \\
\boldsymbol{N} & =\{\sin \Theta(\theta) \cos \varphi, \sin \Theta(\theta) \sin \varphi, \cos \Theta(\theta)\}, \\
F(r, \theta) & =2 \arctan \left\{( \frac { r _ { S } ^ { 2 } } { r ^ { 2 } } ) \left[1+\gamma_{1} \cos \theta\right.\right. \\
& \left.\left.+\gamma_{2} \cos ^{2} \theta+\gamma_{3} \cos ^{3} \theta+\ldots\right]\right\}, \\
\Theta(\theta) & =\theta+\delta_{1} \sin 2 \theta+\delta_{2} \sin 4 \theta+\delta_{3} \sin 6 \theta+\ldots .
\end{aligned}
$$

Here, $F(r, \theta)$ is the profile function of the deformed Skyrmion and $r_{S}, \gamma_{1}, \gamma_{2}, \gamma_{3}, \ldots$ and $\delta_{1}, \delta_{2}, \delta_{3}, \ldots$ are variational (or, respectively, deformation) parameters which are determined from minimizing the Skyrmion energy for a given background baryon density. The density dependence is contained in the medium functionals $\alpha_{s}(\boldsymbol{r})$ and $\alpha_{p}(\boldsymbol{r})$,

$$
\begin{aligned}
& \alpha_{p}(\boldsymbol{r})=1-\frac{4 \pi c_{0} \rho(\boldsymbol{r}) / \eta}{1+g_{0}^{\prime} 4 \pi c_{0} \rho(\boldsymbol{r}) / \eta} \\
& \alpha_{s}(\boldsymbol{r})=1-4 \pi \eta b_{0} \rho(\boldsymbol{r}) / m_{\pi}^{2} .
\end{aligned}
$$

Here $\eta=1+m_{\pi} / m_{N} \sim 1.14$ is a kinematical factor, $m_{N}=$ $938 \mathrm{MeV}$ the mass of the nucleon, $g_{0}^{\prime}=1 / 3$ the Migdal parameter which takes into account the short-range correlations, and $b_{0}=-0.024 m_{\pi}^{-1}$ and $c_{0}=0.21 m_{\pi}^{-3}$ are empirical parameters which can be taken from the analyses of low-energy pion-nucleus scattering data 10]. For more details see Ref. [8]. Since Lorentz invariance is broken at finite density, the time and space derivatives acting on the pion fields have different prefactors. To obtain states with definite spin and isospin, one has to perform adiabatic rotations and quantization of these. In Ref. [8], we have calculated the nucleon mass and other static properties for nucleons inside light, medium and heavy nuclei,

\footnotetext{
2 For the details on the geometry and the justification of this ansatz, see [8].
}

based on realistic nuclear density distributions within the nuclei considered. For example, the decrease of the nucleon mass came out considerably smaller than in earlier studies where uniform baryon matter densities where assumed. It was also shown that the concept of a uniform swelling of nuclear sizes in the medium is too simple to be a realistic picture, in fact the modifications for the baryon matter distribution within a nucleon or the scale changes of the various electromagnetic radii all turned out to be different. In this paper, we extend these considerations to the nucleons form factors at small and intermediate momentum transfer.

\subsection{Electromagnetic form factors}

The electric and magnetic form factors of the nucleon are defined through the expressions

$$
\begin{aligned}
G_{E}\left(q^{2}\right) & =\int d^{3} r e^{i \boldsymbol{q} \cdot \boldsymbol{r}} j^{0}(r), \\
G_{M}\left(q^{2}\right) & =m_{N} \int d^{3} r e^{i \boldsymbol{q} \cdot \boldsymbol{r}}[\boldsymbol{r} \times \boldsymbol{j}(r)],
\end{aligned}
$$

where $q^{2}$ is the momentum transfer squared, $j^{0}$ and $\boldsymbol{j}$ correspond to the time and the space components of the properly normalized sum of the baryonic current $B_{\mu}$ and the third component of the isovector current $\boldsymbol{V}_{\mu}$, i.e.

$$
\begin{aligned}
B_{\mu} & =\frac{1}{24 \pi^{2}} \varepsilon_{\mu \nu \alpha \beta} \operatorname{Tr} L^{\nu} L^{\alpha} L^{\beta} \\
V_{\mu}^{(3)} & =-\frac{i F_{\pi}^{2}}{16} C_{\mu} \operatorname{Tr} \tau_{3}\left(L_{\mu}+R_{\mu}\right) \\
& +\frac{i}{16 e^{2}} \operatorname{Tr} \tau_{3}\left\{\left[L_{\nu},\left[L_{\mu}, L_{\nu}\right]\right]+\left[R_{\nu},\left[R_{\mu}, R_{\nu}\right]\right]\right\}, \\
R_{\mu} & =U \partial_{\mu} U^{+} ; \quad C_{\mu}=\left\{\begin{array}{c}
1, \mu=0 \\
\alpha_{p}, \mu=1,2,3
\end{array}\right.
\end{aligned}
$$

with $\varepsilon_{\mu \nu \alpha \beta}$ the totally antisymmetric tensor in four dimensions and $\mathrm{Tr}$ denotes the trace in $\mathrm{SU}(2)$ flavor space. Evaluating these current operators between appropriate nucleon states as described in [8], one obtains the electromagnetic form factors of the nucleon. For the problem at hand, it is advantageous to expand the plane wave factor in the expressions Eqs. (8),

$$
e^{i \boldsymbol{q} \cdot \boldsymbol{r}}=4 \pi \sum_{l=0}^{\infty} \sum_{m=-l}^{l} i^{l} Y_{l}^{m}\left(\theta_{q}, \varphi_{q}\right) Y_{l}^{m *}\left(\theta_{r}, \varphi_{r}\right) j_{l}(q r)
$$

in terms of spherical harmonics $Y_{l}^{m}$ and spherical Bessel functions $j_{l}$. In this way we get the final expression for the electromagnetic form factors

$$
G_{a}^{b}\left(q^{2}\right)=\sum_{l} i^{l} \sqrt{2 l+1} P_{l}\left(\cos \theta_{q}\right) G_{a}^{b, l}\left(q^{2}\right)
$$

where the label $a$ stands either for electric $(E)$ or magnetic $(M)$ form factors, $b$ stands either for isoscalar $(S)$ or 
isovector $(V)$ form factors, and the $P_{l}$ are Legendre polynomials. After angular integration in momentum space ${ }^{3}$, the moduli of the form factors satisfy the simple rule

$$
\int d \Omega_{q}\left|G_{a}^{b}\left(q^{2}\right)\right|^{2}=4 \pi \sum_{l=0}^{\infty}\left(G_{a}^{b, l}\left(q^{2}\right)\right)^{2}
$$

The corresponding partial form factors have the form

$$
\begin{aligned}
& G_{E}^{S, l}\left(q^{2}\right)=-\frac{\sqrt{2 l+1}}{\pi} \int_{0}^{\infty} r^{2} d r \int_{0}^{\pi} \sin \theta d \theta \\
& \times\left\{j_{l}(q r) P_{l}(\cos \theta) F_{r} \Theta_{\theta} \frac{\sin ^{2} F}{r^{2}} \frac{\sin \Theta}{\sin \theta}\right\}, \\
& G_{E}^{V, l}\left(q^{2}\right)=\frac{\sqrt{2 l+1} \pi}{4 I_{\Omega \omega}^{(33)}} \int_{0}^{\infty} r^{2} d r \int_{0}^{\pi} \sin \theta d \theta \\
& \times\left\{j _ { l } ( q r ) P _ { l } ( \operatorname { c o s } \theta ) \operatorname { s i n } ^ { 2 } \Theta \frac { \operatorname { s i n } ^ { 2 } F } { r ^ { 2 } } \left[F_{\pi}^{2} r^{2}\right.\right. \\
& \left.\left.+\frac{4}{e^{2}}\left(r^{2} F_{r}^{2}+F_{\theta}^{2}+\Theta_{\theta}^{2} \sin ^{2} F\right)\right]\right\} \text {, } \\
& G_{M}^{S, l}\left(q^{2}\right)=-\frac{\sqrt{2 l+1} m_{N}}{4 \pi I_{\Omega \omega}^{(33)}} \int_{0}^{\infty} r^{2} d r \int_{0}^{\pi} \sin \theta d \theta \\
& \times\left\{j_{l}(q r) P_{l}(\cos \theta) F_{r} \Theta_{\theta} \sin ^{2} F \sin \Theta \sin \theta\right\}, \\
& G_{M}^{V, l}\left(q^{2}\right)=\frac{1}{3} \sqrt{2 l+1} \pi m_{N} \int_{0}^{\infty} r^{2} d r \int_{0}^{\pi} \sin \theta d \theta \\
& \times\left\{j _ { l } ( q r ) P _ { l } ( \operatorname { c o s } \theta ) \operatorname { s i n } ^ { 2 } \Theta \frac { \operatorname { s i n } ^ { 2 } F } { r ^ { 2 } } \left[F_{\pi}^{2} r^{2} \alpha_{p}\right.\right. \\
& \left.\left.+\frac{4}{e^{2}}\left(r^{2} F_{r}^{2}+F_{\theta}^{2}+\Theta_{\theta}^{2} \sin ^{2} F\right)\right]\right\} \text {, }
\end{aligned}
$$

where $F_{r}=\partial F / \partial r, F_{\theta}=\partial F / \partial \theta, \Theta_{\theta}=\partial \Theta / \partial \theta$ are partial derivatives and

$$
\begin{aligned}
I_{\Omega \omega}^{(33)} & =\frac{\pi}{4} \int_{0}^{\infty} d r \int_{0}^{\pi} \sin \theta d \theta\left\{\sin ^{2} \Theta \sin ^{2} F\right. \\
& \left.\times\left[F_{\pi}^{2} r^{2}+\frac{4}{e^{2}}\left(r^{2} F_{r}^{2}+F_{\theta}^{2}+\Theta_{\theta}^{2} \sin ^{2} F\right)\right]\right\}
\end{aligned}
$$

is a moment of inertia. The details of the quantization procedure to obtain states with good spin and isospin quantum numbers from the deformed topological soliton are spelled out in [8].

The nucleon form factors are defined as

$$
G_{E, M}^{\left(\begin{array}{c}
p \\
n
\end{array}\right)}\left(q^{2}\right)=\frac{1}{2}\left(G_{E, M}^{S}\left(q^{2}\right) \pm G_{E, M}^{V}\left(q^{2}\right)\right) .
$$

\footnotetext{
3 We note that in the present case the incoming beam direction is in general not the $z$-direction of the coordinate system, since the latter is fixed by the direction of $\mathbf{R}$.
}

We note that the isovector magnetic form factor explicitly depends on the medium functional $\alpha_{p}(r, \theta, R)$. We now want to consider the possible modifications of these form factors within ${ }^{4} \mathrm{He}$. As in the previous work [8] the density is parametrized as

$$
\rho(r)=\left(\frac{3}{4}\right) \frac{2}{\pi^{3 / 2} r_{0}^{3}}\left[1+\frac{A-2}{3}\left(\frac{r^{2}}{r_{0}^{2}}\right)\right] \exp \left\{-\frac{r^{2}}{r_{0}^{2}}\right\},
$$

where the prefactor $3 / 4$ accounts for the fact that we single out one nucleon from the background of the others. The parameter $r_{0}=1.31 \mathrm{fm}$ corresponds to ${ }^{4} \mathrm{He}$ [11]. We are now in the position to study the nucleons' electromagnetic form factors within this light nucleus.

\section{Results and discussion}

Our input parameters are the same as in the Ref. [8], i.e. we use $F_{\pi}=108 \mathrm{MeV}$ and $e=5.265$ (to fit the nucleon and the delta mass). Before discussing any possible medium modifications, we have to consider the electromagnetic form factors in free space. In Fig. I we show the charge form factors of the proton and the neutron in comparison to the dispersion-theoretical results of Ref. [12] and also to the recent Galster-like parameterization of $G_{E}^{n}\left(q^{2}\right)[13$

$$
G_{E}^{n}\left(q^{2}\right)=-\frac{\mu_{n} \tau}{1+3.4 \tau}\left(1+\frac{q^{2}}{0.71 \mathrm{GeV}^{2}}\right)^{-2}
$$

with $\tau=q^{2} / 4 m_{N}^{2}$. The model predictions are in fair agreement with the data, more precisely with the phenomenological fits. A similar statement holds for the momentum dependence of the proton and neutron magnetic form factors, not shown here. Note that we display the form factors only for momentum transfer squared $q^{2} \leq 0.6 \mathrm{GeV}^{2}$ for two reasons. First, the model does not contain vector mesons which start to be relevant at a typical scale $m_{\rho}^{2}=(0.77 \mathrm{GeV})^{2} \simeq 0.6 \mathrm{GeV}^{2}$ (for a review see 14]) and second, boost effects can not be completely ignored any more for these momentum transfers (see e.g. [15] for a discussion on this point). The magnetic moments come out too small, as it is well-known in such type of models. We have $\mu_{p}=1.93$ and $\mu_{n}=-1.20$ in units of nuclear magnetons. Note, however, that the ratio $\left|\mu_{p} / \mu_{n}\right|=1.61$ is close to the empirical value of 1.46 . Other static properties are given in [8].

We now consider the medium modifications due to the finite baryon density within the ${ }^{4} \mathrm{He}$ nucleus. In Table 1 we give the modifications of the nucleon mass, of the proton and neutron magnetic moments and of the variational parameter $r_{S}$ characterizing the Skyrmion size f, cf. Eq. (4), for various distances from the center of the nucleus. We note that the magnetic moments are changed by less than

\footnotetext{
4 Note that we correct for an typographical error that appeared in Eq. (18) of Ref. [8].

5 This variational parameter should not be mixed up with the (isoscalar) r.m.s. radius of the nucleon, see Ref. 8].
} 

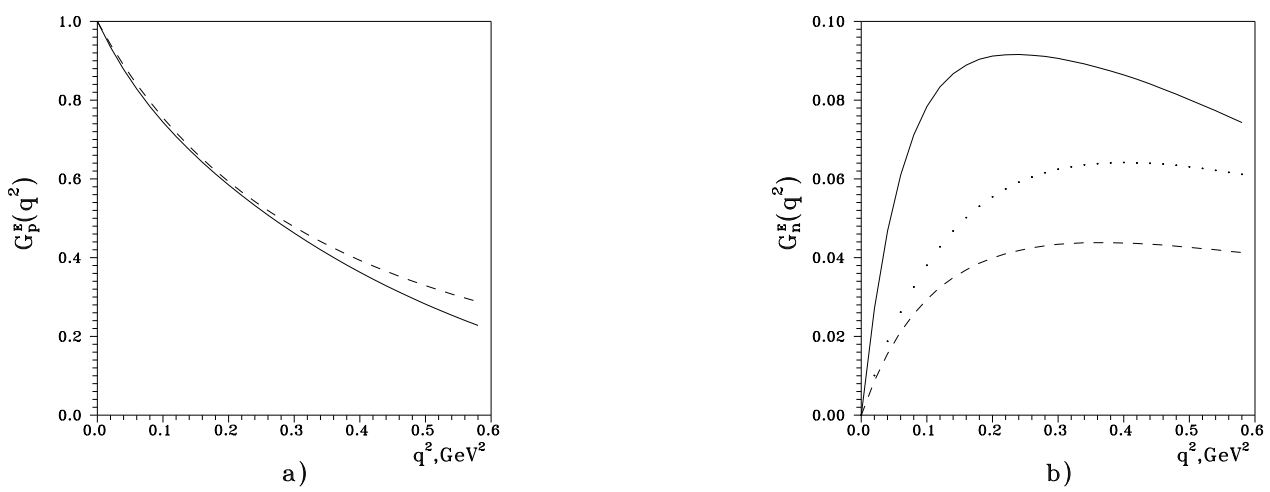

Fig. 1. Electric proton (a) and electric neutron (b) form factor. Solid (dashed) lines: our model (dispersion-theoretical analysis of [1] ). The dotted line in $G_{E}^{n}\left(q^{2}\right)$ represents the parametrization Eq. 19. $)$.

$5.5 \%$. In particular, while we find a mild suppression in the center of the nucleus, at distances above $1 \mathrm{fm}$ the moments are in fact slightly enhanced in magnitude, very different from the behavior of the nucleon mass. The changes in $r_{S}$ are of a similar size. Note, however, that the magnitude of $r_{S}$ decreases monotonically from the center of the nucleus to its surface. These results further demonstrate that the notion of a uniform swelling (or shrinking) of the various nucleon sizes or properties is ruled out by such type of realistic model.

Table 1. Properties of nucleons in ${ }^{4} \mathrm{He}$ and in free space (last row). $R$ is the distance between the centers of the nucleon and the ${ }^{4} \mathrm{He}$ nucleus; $r_{S}$ is a variational parameter as explained in the text.

\begin{tabular}{|c|cccc|}
\hline$R[\mathrm{fm}]$ & $r_{S}[\mathrm{fm}]$ & $\mu_{p}[$ n.m. & $\mu_{n}[$ n.m. & $m_{N} / m_{N}^{\text {free }}$ \\
\hline 0 & 0.627 & 1.883 & -1.137 & 0.817 \\
0.25 & 0.626 & 1.885 & -1.140 & 0.823 \\
0.50 & 0.624 & 1.892 & -1.150 & 0.837 \\
0.75 & 0.621 & 1.907 & -1.168 & 0.860 \\
1.00 & 0.619 & 1.930 & -1.195 & 0.887 \\
1.25 & 0.618 & 1.956 & -1.224 & 0.915 \\
1.50 & 0.617 & 1.975 & -1.245 & 0.941 \\
1.75 & 0.615 & 1.982 & -1.251 & 0.961 \\
2.00 & 0.611 & 1.975 & -1.243 & 0.978 \\
2.25 & 0.607 & 1.962 & -1.230 & 0.988 \\
2.50 & 0.604 & 1.950 & -1.217 & 0.994 \\
\hline- & 0.600 & 1.932 & -1.197 & 1 \\
\hline
\end{tabular}

In Fig. 2 we show the $l=0$ projections of the four nucleon form factors in the medium normalized to their free space values for two different densities, that is various distances from the center of the nucleus. These are $R=0$ (1) fm corresponding to a residual density, cf. Eq. (18), of $0.7(0.55) \rho_{0}$, with $\rho_{0}=0.17 \mathrm{fm}^{-3}$ the nuclear matter density. We note that the medium modifications are small for $q^{2} \leq 0.6 \mathrm{GeV}^{2}$, i.e. for momentum transfers where the model can be considered realistic. These changes stay below $20 \%$ for all form factors. They are in particular small for $G_{E}^{n}\left(q^{2}\right)$, which is often considered to be the most sensitive quantity with respect to such medium modifications. It is interesting to consider the isospin basis. While for the electric case, the isovector piece shows a stronger medium dependence than the isoscalar one, the magnetic isovector and isoscalar form factors exhibit approximately the same suppression for the range of momentum transfers considered here. This latter trend was also found in earlier calculations [2]. The partial form factors for $l \geq 1$, which we do not show, are very small. The results for the proton charge and magnetic form factor are comparable to the ones obtained in [5] in the framework of a quarkmeson model and employing shell like nuclear density distributions, but differ in finer details like the magnitude of the modifications. Note, however, that these authors apply their model to a considerably larger range of momentum transfer. Clearly, our results are also consistent with the limits obtained from electron scattering data based on the $y$-scaling hypothesis [16].

\section{Summary and outlook}

In this paper, we have considered the possible medium modifications of the nucleons electromagnetic form factors in light nuclei, specifically within ${ }^{4} \mathrm{He}$. We have used an extended Skyrme model allowing for deformations of the nucleons immersed in the nuclear medium and applying realistic nuclear matter distributions. We find small medium renormalizations, quite different from the ones obtained using a homogeneous nuclear matter background. In particular, these form factors are not uniformly changed. This indicates that the concept of a uniform swelling or shrinking of the nucleon sizes cannot be maintained. These results are also consistent with the ones found in similar type of models when proper care is taken about the distribution of the nuclear density distributions. Beside the 

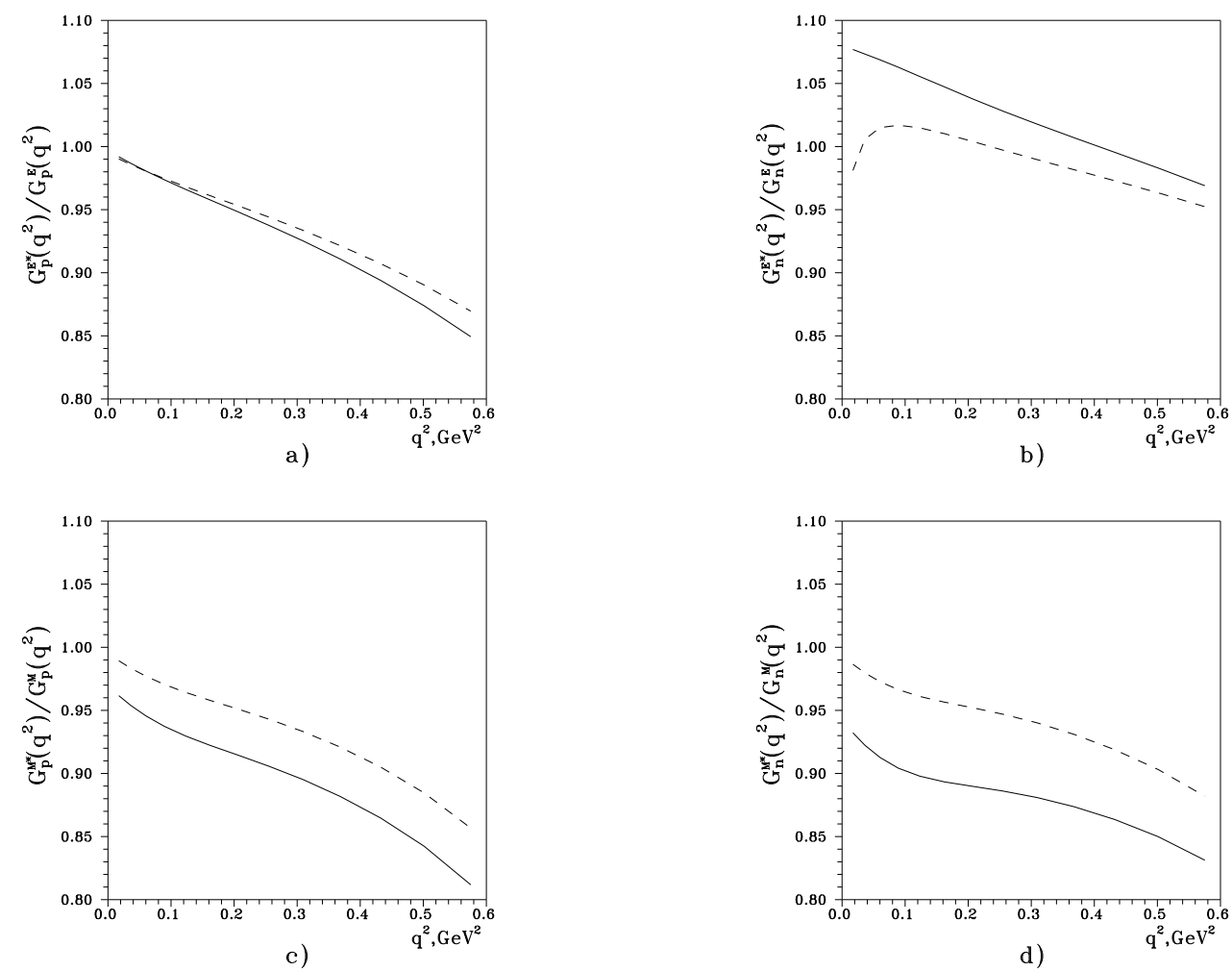

Fig. 2. Form factors normalized to their free space values for two densities within the ${ }^{4}$ He nucleus. The distance of the center of the nucleon from the center of the nucleus is $0 \mathrm{fm}$ (solid lines) and $1 \mathrm{fm}$ (dashed lines). Panels a), b) c) and d) give the proton electric, neutron electric, proton magnetic and neutron magnetic form factor, in order.

small effects considered here, there are also many-body effects that lead to in-medium nucleon changes. These are expected to be significant only in heavier nuclei. From the results presented here, we must conclude that the extraction of the neutron charge form factor from light nuclei at low and intermediate momentum transfer is not sensitive to such effects, given the presently achieved experimental accuracy.

\section{Acknowledgements}

We gratefully acknowledge helpful discussions with Yousuf Musakhanov and Abdulla Rakhimov. We thank Hartmut Schmieden for a useful communication. The work of U.Y. has been supported by an INTAS YS fellowship $\mathrm{N}^{\circ} 00-51$ and SCOPES grant $\mathrm{N}^{\circ} 7 \mathrm{UZPJ} 65677$.

\section{References}

1. L. S. Celenza, A. Rosenthal and C. M. Shakin, Phys. Rev. C 31 (1985) 232.

2. U.-G. Meißner, Phys. Rev. Lett. 62 (1989) 1013; Phys. Lett. B 220 (1989) 1; Nucl. Phys. A 503 (1989) 801.

3. E. Ruiz Arriola, C. V. Christov and K. Goeke, Phys. Lett. B 225 (1989) 22.
4. D. H. Lu, K. Tsushima, A. W. Thomas, A. G. Williams and K. Saito, Phys. Lett. B 441 (1998) 27 arXiv:nucl th/9804009.

5. D. H. Lu, K. Tsushima, A. W. Thomas, A. G. Williams and K. Saito, Phys. Rev. C 60 (1999) 068201 arXiv:nucl th/9807074.

6. M. M. Musakhanov, U. T. Yakhshiev, Z. Kanokov and A. M. Rakhimov, Phys. Atom. Nucl. 62 (1999) 1845.

7. A. M. Rakhimov, M. M. Musakhanov, F. C. Khanna and U. T. Yakhshiev, Phys. Rev. C 58 (1998) 1738 arXiv:nuclth/9609049.

8. U. T. Yakhshiev, M. M. Musakhanov, A. M. Rakhimov, U.G. Meißner and A. Wirzba, Nucl. Phys. A 700 (2002) 403 arXiv:nucl-th/0109008.

9. U.-G. Meißner, J. A. Oller and A. Wirzba, Annals Phys. 297 (2002) 27 arXiv:nucl-th/0109026.

10. T. E. Ericson and W. Weise, Pions and Nuclei (Claredon, Oxford, 1988).

11. A. I. Akhiezer, A. G. Sitenko and V. K. Tartakovsky, Nuclear electrodynamics (Springer, Berlin, 1994).

12. P. Mergell, U.-G. Meißner and D. Drechsel, Nucl. Phys. A 596 (1996) 367 arXiv:hep-ph/9506375; H.-W. Hammer, U.-G. Meißner and D. Drechsel, Phys. Lett. B 385 (1996) 343 arXiv:hep-ph/9604294.

13. C. Herberg et al., Eur. Phys. J. A 5 (1999) 131.

14. U.-G. Meißner, Phys. Rep. 161 (1988) 213.

15. G. Holzwarth, Z. Phys. A 356 (1996) 339 arXiv:hep$\mathrm{ph} / 9606336$.

16. I. Sick, Phys. Lett. B 157 (1985) 13. 